\title{
Valoración del dolor en el anciano
}

\author{
M.P. Sáez López ${ }^{1}$ N. Sánchez Hernández², S. Jiménez Mola³, N. Alonso García ${ }^{2}$ y J.A. Valverde García² \\ ${ }^{1}$ Unidad de Geriatría y ${ }^{2}$ Servicio de Cirugía Ortopédica y Traumatología. Complejo Asitencial de Ávila. \\ Ávila. ${ }^{3}$ Unidad de Geriatría. Complejo Asistencial de León. León
}

Sáez López MP, Sánchez Hernández N, Jiménez Mola S, Alonso García N, Valverde García JA. Valoración del dolor en el anciano. Rev Soc Esp Dolor 2015;22(6):271-274. test diagnósticos y algunas escalas. La elección del instrumento depende del nivel cognitivo, visual, auditivo y capacidad comunicativa de cada paciente.

Palabras clave: Dolor. Anciano. Valoración.

\section{EPIDEMIOLOGÍA}

La prevalencia cruda de cualquier tipo de dolor oscila desde 0 al 93\%. Esta variación puede ser debida al tipo de población, y al método y definición utilizada en los estudios.

La prevalencia de dolor crónico en ancianos que viven en la comunidad es del 25-76\%, mientras que en residencia aumenta hasta 83-93\%. La mayoría de los estudios encuentran mayor prevalencia de dolor en mujeres (1:3 según el estudio EPIDOR) (1,2).

Hay numerosos estudios que evalúan la evolución del dolor con la edad, con resultados inconsistentes, aunque predominando los que concluyen que el dolor aumenta en los más mayores (2).

Tanto el sistema nervioso central como el periférico están afectados por la edad, con reducción de B-endorfina y síntesis de GABA en el tálamo lateral, menor concentración de GABA central y de receptores de serotonina, menor velocidad de procesamiento nociceptivo y menor función de las fibras C y Adelta (3).

No existe clara evidencia de las diferencias en número de nociceptores o aferentes nociceptivos primarios en los ancianos. Sí que se han comunicado algunos hallazgos como descenso de la nocicepción para estímulos de presión, aumento de la tolerancia al dolor cutáneo, reducción de la tolerancia al dolor profundo y tiempo de reacción más largo a la percepción del primer dolor. De estos resultados se puede deducir 
que en el anciano predominan las fibras $\mathrm{C}$ sobre las Adelta, caracterizando el dolor como más lento, sordo y profundo (4).

Según 22 estudios consultados, la localización del dolor más frecuente en ancianos es espalda/columna, piernas (rodilla o cadera) y otras articulaciones, causado por artrosis y lumbalgias crónicas (2), lo que hace del dolor nociceptivo el más frecuente.

Pero también el dolor neuropático representado por neuropatías diabéticas, polineuropatías dolorosas, nutricionales por déficit de vitaminas del complejo B, y especialmente la neuralgia postherpética son frecuentes en el anciano (5). La repercusión del dolor en las personas mayores es aún peor que en el adulto joven. Conlleva aislamiento, depresión, sufrimiento, insomnio, deterioro funcional, caídas, alteración cognitiva, polifarmacia, malnutrición y aumento del coste $(6,7)$, con la consiguiente pérdida de calidad de vida.

Por otro lado, el $60 \%$ de dolor postoperatorio evoluciona a dolor crónico, siendo la intensidad y el mal control el principal factor de riesgo (8).

La Encuesta EPIC (Dolor por cáncer en Europa) sobre prevalencia, tratamiento e impacto del dolor por cáncer, realizada en 12 países europeos, encontró que el dolor es el primer síntoma que induce a los pacientes a buscar atención médica, cuando se les detecta el cáncer. En el 33\% de los europeos, el dolor es el síntoma clave para el diagnóstico de cáncer y hasta el $75 \%$ de los pacientes con cáncer se quejan de dolor asociado con la enfermedad, pero un cuarto de estos pacientes no recibían tratamiento analgésico (9).

En el anciano existen algunas circunstancias que complican tanto la evaluación y diagnóstico del dolor como las posibilidades terapéuticas. Este grupo de edad suele ser excluido de los ensayos clínicos, lo que dificulta el mejor conocimiento de sus problemas. La elevada prevalencia de alteraciones senso-perceptivas, problemas cognitivos y motores limitan la comunicación y por tanto el diagnóstico. Se debe sospechar que sufra dolor ante síntomas tan inespecíficos como confusión, aislamiento y apatía. La pluripatología coexistente ( $40 \%$ de los ancianos tienen al menos 3 enfermedades) y la polifarmacia, predispone a interacciones farmacológicas y mayor toxicidad, sin olvidar tasas de falta de adherencia del $50 \%$ y de automedicación del $80 \%$.

Además, con la edad ocurren múltiples cambios fisiológicos que pueden alterar la farmacocinética y farmacodinamia de los fármacos, complicando aún más el manejo del dolor (10).

\section{CARACTERÍSTICAS DE LA EVALUACIÓN DEL DOLOR EN EL ANCIANO}

Gran parte de la valoración del dolor en el anciano coincide con la realizada a otras edades. Se debe iniciar con una completa historia clínica que contemple características del dolor, forma de inicio, desencadenantes, variaciones, irradiación, registrando las distintas terapias utilizadas y su eficacia. Debe continuarse con una exploración física, para concretar la localización y tipo de dolor. Además, se debe interrogar sobre la repercusión en la vida diaria, a nivel funcional, afectivo y social.

Especial importancia tiene en el anciano la evaluación estandarizada de la capacidad funcional, ya que el dolor condiciona un deterioro de la misma, pudiendo provocar una situación de dependencia a veces irreversible si no se detecta de forma precoz. Esta información puede ayudar al diagnóstico de dolor, ya que con frecuencia los ancianos pierden la movilidad y la autonomía para evitar maniobras dolorosas. También sirve para establecer los objetivos del tratamiento y comprobar la eficacia, incluyendo la recuperación funcional entre dichos objetivos, además de la analgesia.

Existen numerosos instrumentos de evaluación funcional, aunque los más utilizados son el Índice de Lawton y Brody para actividades instrumentales y el Índice de Barthel para actividades básicas.

Además de la información aportada por el paciente, el uso de escalas de valoración aumenta la sensibilidad diagnóstica y facilita el seguimiento del síntoma y su propio tratamiento. El instrumento ideal debe ser sencillo de aplicar y válido para cualquier paciente, incluyendo aspectos sobre la percepción del dolor y estado psicológico del paciente (11).

- La escala analógica visual que mide la intensidad de dolor mediante una línea de $10 \mathrm{~cm}$ de longitud en cuyos extremos figura ausencia de dolor y dolor máximo, suele ser sencilla y fácil de aplicar. De cualquier forma, proporciona más fallos en ancianos con bajo nivel cultural o deterioro cognitivo, déficit motor o en muy mayores. La escala verbal descriptiva, que contempla dolor leve-moderado-severo, puede resultar más fácil de entender para este tipo de pacientes.

- Los resultados obtenidos en escalas pictóricas (de caras) como FPS, se afectan menos en pacientes con demencia o bajo nivel educativo. Cuentan con buenas propiedades psicométricas en personas mayores españolas con bajo nivel cultural porque solo tienen que elegir entre diferentes caras, eliminando el componente verbal.

- Sin embargo, cuestionarios multidimensionales que abarcan aspectos como localización, intensidad del dolor, evolución temporal, repercusión afectiva y funcional como Cuestionario McGill o Brief Pain Inventory, suelen ser difíciles de aplicar y no resultan válidos en deterioro cognitivo, por lo que se reservan para investigación.

\section{VALORACIÓN DEL DOLOR EN PACIENTES CON DEMENCIA}

Los pacientes con demencia, especialmente si es moderada o severa, suelen tener dificultades para expresar el 
dolor, lo que conlleva falta de diagnóstico y de tratamiento. Solo el $16 \%$ de pacientes dementes con patología dolorosa recibían analgésicos en una residencia (12). También pacientes con fractura de cadera y demencia se utiliza 1/3 de la dosis de morfina habitual y el $76 \%$ no tenían tratamiento para el dolor incidental (13).

El tratamiento empírico con paracetamol cada $8 \mathrm{~h}$ pautado en pacientes con demencia moderada-severa en 25 residencias en un estudio randomizado, controlado con placebo consigue mayor interacción social y mejoría del lenguaje y del comportamiento (14).

En pacientes con demencia no se aprecian cambios en el umbral de respuesta al dolor, pero sí en la tolerancia al mismo, es decir, aunque el componente sensitivo está indemne, el componente afectivo sí que se encuentra modificado (15). Existen algunas diferencias en la percepción del dolor, según el tipo de demencia:

Se sabe que la respuesta autonómica al dolor decrece en pacientes con enfermedad de Alzheimer (EA), debido a la localización de las lesiones de la EA (16).

La demencia vascular tiene incrementado el componente emocional del dolor, ya que las lesiones de sustancia blanca incrementan la sensibilidad por desaferentación, lo que se relaciona con la presencia de dolor central (17).

En la demencia frontotemporal se aprecia una reducción del procesamiento y del componente emocional, con mayor tolerancia al mismo (18).

En la demencia de Cuerpos de Lewy se reduce la percepción del dolor y del sufrimiento, con lesiones en la sustancia blanca similares a las de EA pero con mayor alteración en el área para-silviana (19).

El 60-80\% de pacientes con demencia de una residencia sufrían dolor predominantemente de origen osteomuscular, gastrointestinal, genitourinario y por úlceras por presión (20). El dolor de órganos internos o piel es más difícil de evaluar en estos pacientes que el osteomuscular que suele reproducirse con los movimientos. El dolor crónico también es más difícil de detectar que el dolor agudo.

Por tanto, en pacientes con demencia, debemos tener aún más conciencia de la posibilidad de que sufre dolor para mejorar el diagnóstico. Para ello, son útiles los autoinformes y la observación del paciente, que en estos casos se pueden manifestar como vocalizaciones, quejidos, gritos, muecas o gestos faciales, movimientos corporales anormales, alteraciones de conducta, insomnio y delirium. Algunas variables fisiológicas pueden resultar útiles, aunque no específicas, como: TA, frecuencia respiratoria, sudoración palmar, liberación hormonas de estrés, liberación de cortisol y concentraciones de endorfinas (21). También la información de familia y cuidador puede ser útil, aportando mayor valor cualitativo que cuantitativo.

Existen escalas para evaluar el dolor en pacientes con demencia. Cuando esta es moderada se pueden utilizar las escalas descriptivas verbales. Sin embargo, en el grado severo se deben utilizar las que evalúan gestos, verbalizaciones y lenguaje corporal como Doloplus 1, Algoplus 2 y DS-DAT Discomfort Scale-Dementia 3.

Lo cierto es que aunque se dispone de numerosos instrumentos de evaluación, muchos de ellos requieren una validación, así como demostrar su utilidad en la práctica. Además no son capaces de distinguir entre el tipo de dolor, ni su cronología y tampoco contemplan el dolor en diferentes circunstancias (descanso, realización de actividades básicas o movilizaciones).

Las dificultades del reconocimiento del dolor en pacientes con demencia, junto con las barreras existentes sobre el uso de determinados analgésicos, contribuyen a que el personal que les atiende no esté suficientemente preparado para detectarlo y tratarlo adecuadamente. Pero impartirles una adecuada formación y entrenamiento en la evaluación y manejo tanto farmacológico como no farmacológico del dolor, puede mejorar en gran medida su competencia en el abordaje de este problema. Se pueden encontrar recomendaciones prácticas y útiles para el personal de enfermería que trabajan en residencias en la página www.geriatricpain.org. No obstante, el cambio en el manejo pasa por implicar a todo el equipo multidisciplinar que atiende a estos pacientes, con sensibilización, y una formación adecuada (22).

En la tabla I se sugieren las escalas más adecuadas según el grado de deterioro cognitivo $(15,23)$.

TABLA I. ESCALAS DE VALORACIÓN DEL DOLOR EN PACIENTES CON DIFERENTES GRADOS DE DETERIORO COGNITIVO

\begin{tabular}{lll}
\hline \multicolumn{1}{c}{ Nivel cognitivo } & Escala evaluación del dolor & \multicolumn{1}{c}{ Comentario } \\
\hline Intacto & EVA o VAS, NRS, VRS y FPS & 1. NRS y VRS \\
& & Utilizar VAS en vertical \\
Deterioro leve-moderado & VRS, Pain Termometer, FPS & Precisan más explicaciones y más tiempo \\
Deterioro severo & Doloplus-2, Algoplus, DS-DAT & 1. $^{\circ}$ Algoplus por ser más corto \\
\hline
\end{tabular}

VAS: visual analogue scale; NRS: numeric rating scale; VRS: verbal rating scale; FPS: facial pain scale. 


\section{CORRESPONDENCIA:}

María del Pilar Sáez López

Unidad de Geriatría

Complejo Asistencial de Ávila

Av. Juan Carlos I, s/n

05071 Ávila

e-mail: pisalop@gmail.com

\section{BIBLIOGRAFÍA}

1. Gamero F, Gabriel R, Carbonell J, et al. El dolor en las consultas de Reumatología españolas: estudio epidemiológico EPIDOR. Rev Clin Esp 2005;205:157-63.

2. Abdulla A, Adams N, Bone M, Elliott AM, Gaffin J, Jones $\mathrm{D}$, et al.; British Geriatric Society. Guidance on the management of pain in older people. Age Ageing 2013;42(Supl. 1):i1-57. DOI: 10.1093/ageing/afs200.

3. Gibson SJ, Farell MJ. A review of age differences in the neurophysiology of nociception and the perceptual experience of pain. Clinical Journal of Pain 2004;20:227-39.

4. López Mongil R, López Trigo JA. Prevalencia y fisiopatología del dolor crónico en el anciano. En: Guía de buena práctica clínica en Geriatría. Dolor crónico en el anciano; 2012. p. 9-23.

5. Catalá E, Ferrándiz M. Neuralgia postherética. En: Serra Catafau. Tratado de dolor neuropático. Madrid: Ed Médica Panamericana; 2007. p. 369-79.

6. Martinez-Vazquez de Castro J, Torres LM. Prevalencia del dolor postoperatorio. Alteraciones fisiopatológicas y sus repercusiones. Rev Soc Esp Dolor 2000;7:465-76.

7. Leveille SG, Jones RN, Kiely DK, Hausdorff JM, Schmerling RH, Guralnikl JM, et al. Chronic musculoskeletal pain and the occurrence of falls in an older population. JAMA 2009;302:2214-21.

8. Rathmell JP, Wu CL, Sinatra RS, Ballantyne JC, Ginsberg B, Gordon DB, et al. Acute post-surgical pain management: A critical appraisal of current practice regional anesthesia and pain medicine. Reg Anesth Pain Med 2006;31(4 Supl. 1):1-42.

9. Breivik H, Borchgrevink PC, Allen SM, et al. Assessment of pain. Br J Anaesth 2008;101:17-24.

10. Rastogi R, D Meek B. Management of chronic pain in elderly, frail patients: Finding a suitable, personalized method of control. Clinical Interventions in Aging 2013; 8:37-46.
11. J Gutierrez Rodríguez. Clasificación etiopatogénica y valoración. En: Guía de buena práctica clínica en Geriatría. Dolor crónico en el anciano; 2012. p. 25-35.

12. Farrell MJ, Katz B, Helme RD. The impact of dementia on the pain experience. Pain 1996;67(1):7-15.

13. Engle VF, Graney MJ, Chan A. Accuracy and bias of licensed practical nurse and nursing assistant ratings of nursing home resident pain. J Gerontol A Biol Sci Med Sci 2001;56:M405-11.

14. Chibnall JT, Tait RC, Harman B, Luebbert RA. Effect of acetaminophen on behavior, well-being, and psychotropic medication use in nursing home residents with moderate-to-severe dementia. J Am Geriatr Soc 2005;53(11): 1921-9.

15. Alaba J, Arriola E, Navarro A, González MF, Buiza C, Hernández C, et al. Demencia y dolor. Rev Soc Esp Dolor 2011;18(3):176-86.

16. Benedetti F, Arduino C, Vighetti S, Asteggiano G, Tarenzi L, Rainero I. Pain reactivity in Alzheimer patients with different degrees of cognitive impairment and brain electrical activity deterioration. Pain 2004;111(1-2):22-9.

17. Mori E. Impact of subcortical ischemic lesions on behavior and cognition. Ann NY Acad Sci 2002;977:141-8.

18. Bathgate D, Snowden JS, Varma A, Blackshaw A, Neary D. Behaviour in frontotemporal dementia, Alzheimer's disease and vascular dementia. Acta Neurol Scand 2001;103: 367-78.

19. Burton EJ, Karas G, Paling SM, Barber R, Williams ED, Ballard CG, et al. Patterns of cerebral atrophy in dementia with Lewy bodies using voxel-based morphometry. Neuroimage 2002; 17:618-30.

20. Corbett A, Husebo B, Malcangio M, et al. Assessment and treatment of pain in people with dementia. Nat Rev Neurol 2012;8(5):264-74.

21. Gagliese 1. Assesmento of pain in elderly people. In: Turck DC, Melzack R editors. Handbook of Pain Assesement. New York-London: The Guilford Press; 2001. P. 119-33.

22. Hadjistavropoulos T, Janzen Claude JA, Hadjistavropoulos H, Marchildon GP, Kaasalainen S, Gallagher R, et al. Stakeholder opinions on a transformational model of pain management in long-term care. J Gerontol Nurs 2011;37(7): 40-51.

23. Torvik K, Kaasa S, Kirkevold $\varnothing$, Saltvedt I, Hølen JC, Fayers P, et al. Validation of Doloplus-2 among nonverbal nursing home patients-an evaluation of Doloplus-2 in a clinical setting. BMC Geriatr 2010;10:9. 